RTW trajectories were identified: full-time RTW $(n=645)$, late or no RTW $(n=114)$, early and progressive RTW $(n=134)$, full time RTW before early retirement $(n=46)$. BCS that had a late or did not RTW within the five years following their diagnostic were associated with an increased risk of long-term depressive symptoms (OR : 2.73, 95\% CI [1.47-5.04]).

Conclusion This study highlighted that a late or absence of RTW within the 5 years after BC was associated with poorer long-term psychosocial factors and confirmed the potential of using sequence analysis to capture the multi-state aspect of RTW trajectories.

\section{0-320 RETURN TO WORK AND JOB LOSS FOLLOWING HIP REPLACEMENT: FINDINGS FROM TWO LONGITUDINAL COHORTS}

${ }^{1}$ Elena Zaballa, Georgia Ntani, E Clare Harris, Anne Lübbeke-Wolff, Karen Walker-Bone, Nigel K Arden, Cyrus Cooper. 'University of Southampton, United Kingdom

\subsection{6/OEM-2021-EPI.133}

Introduction People are increasingly encouraged to work to older ages, thus returning to and staying in work is an important outcome for younger arthroplasty recipients.

Objectives We examined the impact of physically-demanding occupational activities on the risk of leaving a job because of difficulties with the replaced hip.

Methods A survey was mailed to 1,457 unilateral THA recipients of working age (18-64 years) from the Geneva Hip Arthroplasty Registry and the Clinical Outcomes in Arthroplasty study. People were eligible if they had received their arthroplasty 5 years before. We collected demographic data, time to reach best function and postoperative recreational activities. For each job held postoperatively, participants self-reported exposure to activities that loaded the joint (standing, walking, kneeling/squatting, climbing ladders, lifting, digging). The risk of job loss in relation to occupational activities was calculated using Cox regression models adjusting for age at operation, sex, body mass index, time to reach best post-operative function, cohort and follow-up.

Results In total 514 of 817 respondents (57\% response rate) resumed work post-arthroplasty. Amongst these (206 men and 205 women), 411 self-reported usable occupational information. The median follow-up post-THA was 7.5 years (IQR 6.2 -12.1). Adjusted models showed that there was an increased risk of exiting work post-arthroplasty because of problems with the replaced hip were increased if workers were exposed to: standing $>4$ hours/day (HR:3.81, 95\%CI 1.62-8.96); kneeling/squatting (HR:95\%CI 3.32, 1.46-7.55) and carrying/lifting $>10 \mathrm{~kg}$ (HR:5.43, 95\%CI 2.29-12.88) compared with those who did not.

Conclusion Certain types of occupational activities may hamper job retention following THA. Our results, although subject to replication, suggest that some types of more physicallydemanding work may be more challenging to continue posthip arthroplasty. There may be a role for focussed rehabilitation or career advice or re-deployment of people in some types of jobs.

\section{Sex and Gender}

\section{0-91 WORKPLACE INJURIES AND ILLNESS: WHAT DIFFERENCE DO SEX AND GENDER MAKE? A SYSTEMATIC REVIEW}

${ }^{1}$ Aviroop Biswas, Shireen Harbin, Emma Irvin, Heather Johnston, Momtaz Begum, Maggie Tiong, Dorothy Apedaile, Mieke Koehoorn, Peter Smith. 'Institute for Work \& Health, Canada

\subsection{6/OEM-2021-EPI.134}

Introduction As policymakers become increasingly interested in taking gender/sex differences into account in their primary prevention approaches to occupational health and safety, there is a need to summarize the existing research evidence to find where health outcome differences associated with occupational hazard exposures exist between men and women.

Objective To understand similarities and differences between men and women in health outcomes related to occupational hazard exposures, across different occupations and in the same occupations.

Methods A systematic literature review was conducted on peer-reviewed prospective epidemiological studies published from 2009 to 2019, with no language restrictions. The methodological quality of studies was assessed, with medium to high scoring studies included in the evidence synthesis. Selected studies were qualitatively analysed and compared according to the magnitude of health risks for men and women for each occupational exposure category across occupations and in the same occupations.

Results 105 studies were reviewed. Across occupations, men were at higher risk of kidney disease from occupational heat stress, and injury/disability from physical and biological/chemical hazards. Women were at higher risk of injury/disability, musculoskeletal disorders from biomechanical strain, and poor mental health from workplace stress. In the same occupations, women in the healthcare industry were at greater risk of cancers and injury compared to men in the same jobs. Both men and women exposed to work stress in the same white collar and blue jobs were at risk of injury and heart disease. Men and women working in chemical manufacturing were at risk for different cancers.

Conclusion Men and women have different health risks from exposures to occupational hazards, with differences not solely due to the gendered distribution of occupations. These results may be useful to policy makers seeking to reduce gender inequalities in occupational health, and to researchers wishing to analyse these determinants in greater depth.

\section{0-124 MEN AND WOMEN AT WORK IN CANADA, 1991-2016}

${ }^{1}$ Emma Quinn, Andrew Harper, Cheryl Peters, Ela Rydz, Peter Smith, Mieke Koehoorn. 'University of British Columbia, Canada

\subsection{6/OEM-2021-EPI.135}

Introduction Women's increased labour force participation in Canada is a well-known trend over the past 40 years, and there is a perception that the gendered division of the labour force has decreased over time. 
Objectives The study objective was to document the division of occupations by sex/gender in Canada and to examine the trends since 1991. The evidence is intended to inform occupational health and safety policies and procedures by including issues of sex/gender as part of the discourse on risk prevention, where warranted.

Methods Data obtained from the last six Canadian Censuses of Population (1991-2016) were analyzed and descriptive statistics were used to examine the labour force composition within various resolutions of the National Occupational Classification (NOC) codes by sex/gender. GEE Poisson regression models were used to generate time- and occupation-adjusted estimates for incidence rate ratios with 95\% confidence intervals for sex/gender differences in the labour force. Highly divided occupations were defined as those with $75 \%$ or more men or women.

Results Of the 500 4-digit occupational categories representing 2,892 data points over the 25-year period, 58\% were highly divided, with more than three-quarters of these incidences being for male-dominated occupations, with less than onequarter being for female-dominated occupations. GEE analyses of these occupation groups within broad occupational groups suggested relative stability in the gendered nature of occupations over time, with a statistically significant reduction in the proportion of highly divided occupations only observed among occupations broadly grouped within natural and applied sciences.

Conclusion The Canadian workforce remains highly sexed/gendered. While the distribution of men and women within occupations is driven by complex factors, our inquiry into these found that systems of gender essentialism, organizational hierarchies that favour men, and labour markets that are changeresistant remain challenges.

\section{0-250 ASSOCIATION OF CHILD NEURODEVELOPMENTAL AND BEHAVIOURAL PROBLEMS WITH MATERNAL UNEMPLOYMENT IN A POPULATION-BASED BIRTH COHORT}

'Joana Amaro, Maja Popovic, Milena Maule, Ingrid Sivesind Mehlum, Raquel Lucas, Raquel Costa. 'Institute of Public Health, University of Porto, Portugal

\subsection{6/OEM-2021-EPI.136}

Introduction Mothers typically adjust their work participation in order to meet family needs, including their children's wellbeing. However, there is scarce evidence on the impact of children's poor mental health on maternal employment in less affluent European economies, where the dual-earner family structure is often necessary to make ends meet.

Objectives To estimate associations between suspected and diagnosed neurodevelopmental and behavioural problems in 7 year-old children and maternal unemployment at child ages 7 and 10 , in a Portuguese birth cohort.

Methods We evaluated 5754 mother-child pairs of the population-based birth cohort Generation XXI. Data on child suspected and diagnosed neurodevelopmental and behavioural problems, namely learning, attention and language problems, externalizing behaviours, developmental delay, autism spectrum disorders, and other neurodevelopmental problems, were retrieved at 7 years of age using caregivers' reports. Maternal employment status was collected at child age 7 and 10. Robust Poisson regression models were used to calculate prevalence ratios (PRs) and 95\% confidence intervals (CIs).

Results After adjustment for maternal age, education, being a single mother, singleton/multiple pregnancy, having children under the age of 6 years, household monthly income and maternal mental disorder diagnosis, women were more likely to be unemployed at child age 10 if the child had, up to age 7 , any of the following suspected problems: externalizing behaviours ( $\mathrm{PR}=1.20$, 95\% CI [1.03-1.40]), developmental delay $(\mathrm{PR}=1.46$, 95\% CI [1.12-1.91]), or an autism spectrum disorder $(\mathrm{PR}=1.62,95 \% \mathrm{CI}$ [0.99-2.65]). When the exposure was restricted to clinically diagnosed disorders, the magnitude of associations remained similar, although estimates were less precise. Associations with unemployment were stronger when the child was $10 \mathrm{com}$ pared to when the child was 7 .

Conclusion Having a child with a developmental or behavioural problem or an autism spectrum disorder at age 7 was associated with maternal unemployment three years later, placing families at a higher risk of financial vulnerability.

\section{0-378 THE ROLE OF GENDER IN THE ASSOCIATION BETWEEN SEXUAL HARASSMENT AT WORK AND SUICIDAL IDEATION: A STUDY WITH CIVIL SERVANTS FROM A MIDDLE-INCOME COUNTRY}

${ }^{1}$ Fernando Feijó, Inaê Dutra Valério, Débora Gräf, Maria del Pilar Flores-Quispe, Anaclaudia Fassa. 'Federal University of Bahia, Brazil

\subsection{6/OEM-2021-EPI.137}

Introduction Sexual harassment is associated with several mental health problems, including suicidality. Despite its terrible consequences for individuals, it is frequently an invisible occupational risk. Women are more likely to be harassed than men. However, little is known about gender differences and effect modification on this association.

Objectives To analyse the association between sexual harassment at work and suicidal ideation(SI) in civil servants from a state in Southern Brazil, investigating gender differences and interaction.

Methods This is a cross-sectional study with data from a web survey with employees from the Federal Judicial Court in Southern Brazil. Sexual harassment was evaluated using a structured questionnaire with specific questions about harassment and violence. SI was assessed using the Self-Reporting Questionnaire (SRQ-20). Poisson regression was used to test associations of interest and estimate prevalence ratios (PR). Stratified analyses by sex were performed.

Results 1,636 workers provided complete information. The prevalence of self-reported sexual harassment was 5.6\%, $2.7 \%$ among males and $8.2 \%$ among females. The prevalence of SI was $4.2 \%, 5.0 \%$ in males and $3.5 \%$ in females. Among harassed men, the prevalence of SI was $27.3 \%$. Sexual harassment was positively associated with SI after adjustment for age and educational level(PR 2.31; 95\%CI 1.134.72). In the analysis stratified by sex, the association remained positive only in males $(\mathrm{PR}=4.70$; 95\%CI 1.90 11.66), after adjustment for age, educational level and personality traits.

Conclusions Gender play an important role in the association between sexual harassment and suicide. Approaching the understanding of gender to the measured variable of sex, the 